Review article

\title{
Presbyopia and the aging eye: Existing refractive approaches and their potential impact on dry eye signs and symptoms
}

\author{
Lafosse $\mathrm{E}^{\mathrm{a}, \mathrm{b}}$, Wolffsohn JS ${ }^{\mathrm{b}}$, Talens-Estarelles $\mathrm{C}^{\mathrm{a}}$, García-Lázaro $\mathrm{S}^{\mathrm{a}, *}$ \\ ${ }^{a}$ Optometry Research Group, Department of Optics \& Optometry \& Vision Sciences, University of Valencia, Valencia, Spain \\ ${ }^{\mathrm{b}}$ Ophthalmic Research Group, School of Life and Health Sciences, Aston University, Birmingham, UK
}

\section{A R T I C L E I N F O}

\section{Keywords:}

Presbyopia

Dry eye

Contact lenses

Refractive surgery

Cornea

\begin{abstract}
A B S T R A C T
Every part of the human body is subject to aging, including the eye. An increased prevalence of dry eye disease with age is widely acknowledged. Aging threatens ocular surface homeostasis, altering the normal functioning of the lacrimal functional unit and potentially leading to signs and symptoms of dry eye. Additional age-related processes take place within the crystalline lens, leading to presbyopia and cataractogenesis. Correction strategies for presbyopia and cataracts may directly or indirectly challenge the ocular surface. Contact lenses disturb the normal structure of the tear film and can interact negatively with the ocular surface, further deteriorating an already unbalanced tear film in presbyopes, however, newer contact lens designs can overcome some of these issues. Moreover, cataract and corneal refractive surgeries sever corneal nerves and disrupt the corneal epithelium and ocular surface, which can influence surgical outcomes and aggravate dryness symptoms in older age groups. This review summarises the current understanding of how the invasive nature of contact lens wear and cataract and refractive surgery influence signs and symptoms of ocular dryness in an aging population.
\end{abstract}

\section{Introduction}

The ability of the ocular surface to respond adequately to environmental challenges depends on the appropriate detection of sensations; this involves the transmission of the stimulated signal to the brain and the generation of a response, that modulates secretory function [1] and local immunity [1,2]. Any disturbance to one of the three steps of this closed loop could trigger an inappropriate response and alter the compensatory mechanisms taking place at the ocular surface.

The lacrimal functional unit (LFU) is a set of anatomical structures, whose harmonious functioning maintains tear film (TF) osmolarity within narrow limits [2]. The LFU is composed of: the lacrimal glands (LG), meibomian glands (MGs), the ocular surface (cornea and conjunctiva) and the nerves that connect them [3]. Likewise, the precorneal TF behaves as a single dynamic functional unit with different compartments. Tear dysfunction, more common with ageing, results from degenerative or pathologic processes of one or more components of the LFU, potentially leading to signs and symptoms of dry eye disease (DED) [4]. Every part of the human body is subject to aging and the LFU is no exception: LG, the eyelid area, MGs and conjunctiva are affected in terms of their structure and function over the life span [5-9]. Increasing age challenges ocular surface homeostasis by inducing drastic changes to the LFU: the LG undergoes histologic changes leading to pathological processes (for example a decrease in mass, atrophy of lacrimal ducts and acini, lymphocyte infiltration) and to a diminution in lacrimal secretion $[5,6]$. Furthermore, eyelids also undergo age-related changes that could promote signs and symptoms of dryness among which are: increased lid laxity [7] and MGs atrophy [8]. Conjunctivochalasis, another age-related disorder, is characterized by the presence of folds on the conjunctiva [9] which are known to impact tear meniscus distribution along the eyelid and thus tear meniscus parameters [10], and could play a role in DED onset and perpetuation.

According to the Report of the Tear Film and Ocular Surface (TFOS) [11], an increased prevalence of DED with age is widely acknowledged $[12,13]$. Based on estimates of the number of people over 60 years of age (2 billion people by the year 2050) [14] and an approximate prevalence of $25 \%$ for the disease, 500 million people will suffer from dry eye globally just in this age group [15]. Hence the burden to society will be immense.

Over and above the age-related changes already mentioned taking place in the LFU, two additional visual impairing processes take place within the eye's crystalline lens, leading to presbyopia and cataractogenesis respectively.

With age, the crystalline lens progressively loses its ability to change shape, and the eye's focusing range reaches a point were near vision is insufficient to satisfy an individual's requirements [16]. Symptoms of

\footnotetext{
* Corresponding Author.

E-mail address: santiago.garcia-lazaro@uv.es (S. García-Lázaro).
} 
presbyopia appear around 45 years of age [17], although other elements may influence its onset and progression (such as pupil size, disease, medications and trauma) [18]. Specifically, presbyopia affected 1.3 billion people worldwide in 2011 [19], and up to 2 billion people in 2012 [20]. In this regard, with increasing life expectancies, this trend is expected to keep on rising [21].

Additionally, according to the World Health Organization (WHO), cataract is the leading cause of blindness [22] and the consequent loss of useful vision is expected to affect 16 million people worldwide [23]. Cataractogenesis encompasses a broad spectrum of changes regarding biochemical processes taking place in the crystalline lens leading to an alteration in water balance, proteins, vitamins and enzymes, being responsible for a progressive loss of lens transparency [24]. In this respect, aging is by far the major risk factor for its onset[22,23].

Nowadays, various refractive means exist to correct presbyopia. In this context, contact lenses (CLs) with different optical profiles (monovision, alternating images, simultaneous images) can be used for the purpose. However, once inserted onto the ocular surface, CLs disturb the normal structure of the TF. Refractive surgery is another option available, but due to its potential to sever corneal nerves and disrupt the corneal epithelium, tends to disrupt the ocular surface and worsen or induce signs and symptoms of dryness.

CLs and corneal refractive strategies along with cataract surgery directly or indirectly interact with the ocular surface, threatening its homeostasis. These interactions are particularly relevant for the aging eye, when degenerative processes occurring in the LFU may potentially lead to tear dysfunction. In this regard, it is relevant for the clinician to understand the potential ocular surface and dryness-related outcomes of each refractive correction or procedure in older adults.

Accordingly, this review summarises the current understanding of how the invasive nature of contact lens wear and cataract and refractive surgery influence signs and symptoms of ocular dryness in an aging population.

\section{Corneal innervation and physiological role}

Nerve fibers enter the cornea in the middle third of the stroma and then course through the superior layers forming a plexus in the subBowman's layer that densely innervates central cornea [25]. Corneal nerves terminate in the wing cell layer of the epithelium after penetrating Bowman's layer and losing their myelin sheath. These nerves are key to ocular surface homeostasis, constantly adapting the ocular surface response to environmental challenges. Free nerve endings, more precisely the intra epithelial sensory terminals, are excited in response to different stimuli (mechanical forces, cooling and increased osmolarity) [26] giving rise to afferent impulses that travel along the ophthalmic branch of the trigeminal nerve to the central nervous system [27]. These allow for the detection of potentially damaging stimuli and the induction of defensive reflexes [11] provided by the efferent pathways such as lacrimation, blinking and regulation of different LG secretions [11].

Furthermore, nerve bundles play an important trophic role for the corneal epithelium (involved in nutrition processes) and modulate immune responses and wound healing processes [28]. The different surgical procedures described later on, all impact upon corneal tissue (as an entry porthole or as part of the refractive correction). As such, corneal integrity may be jeopardized, leading to alterations of the closed loop described above and to DED.

\section{Eye surgery and dry eye}

\subsection{Cataract surgery}

Cataract surgery is the most commonly performed elective surgery with an estimated 19 million procedures performed worldwide in 2013-2014 [29,30]. The WHO has forecast a significant increase of this surgery by the year 2020 (estimated 32 million procedures a year) as the number of people over 65 is expected to increase significantly [31].

Firstly, before any surgical treatment, biometric measurements are required in order to calculate the power of the intraocular lens (IOL) to be implanted. The accuracy of these measurements, and hence the postsurgical refractive outcomes, are influenced by TF quality and stability [32,33].

Risk factors for dry eye following cataract surgery regardless of the technique used are well known, but the mechanisms through which they induce dry eye are yet to be established. The following risk factors could be related to the disruption of corneal nerves and harm to the epithelium through the surgical procedure: eyedrops containing active agents/preservatives affecting the epithelium pre-, peri- and post-surgery $[34,35]$; forced opening of the eyelid with the blepharostat prevents normal blinking, thus an even distribution of the TF across the ocular surface [36]; long microscopic light exposure times, which may lead to thermal damage [34]; repeated irrigation of the ocular surface may impact goblet cell density and further impact TF stability [34-37]; and incision location and accuracy, that will be discussed later on in this manuscript. Consistently, studies agree that the surgical procedure increases signs and symptoms of ocular dryness [34,36], with neurogenic inflammation and epithelial (corneal and conjunctival) damage induced by the surgery, being the principal factors acting as DED triggers [38].

Additionally, surgery-induced corneal nerve damage impairs corneal sensitivity [11]. This further affects blink rate and reflex-induced lacrimal secretion [39], which eventually leads to TF instability and increased osmolarity [39]. Tear hyperosmolarity induces epithelial cell hyperosmolarity leading to the liberation of pro-inflammatory CKs, inducing cellular apoptosis and corresponding ocular surface staining.

Previous studies have investigated the pathophysiology of dry eye after cataract surgery [36,38,40-45] (Table 1). These have demonstrated a significant increase in dry eye signs and symptoms, including worse Ocular Surface Disease Index (OSDI) questionnaire scores [36,38], Tear Breakup Time (TBUT) [36,38,40,43,44], Schirmer test $[36,38,40]$, corneal and conjunctival staining $[38,40]$, Tear Meniscus Height (TMH) [36], and corneal sensitivity [41,42,45] until about 2-3 months postoperatively.

Nowadays, a newer technique, called Femtosecond Laser Assisted Cataract Surgery (FLACS) can be used [46] to create the required corneal incisions, capsulotomy and fragmentation of the lens prior to phacoemulsification. This technique is far more accurate than mechanical devices and improved safety and clinical outcomes are expected [46-49]. One drawback of this technique, however, is the pressure to which the peri-limbic conjunctiva is subjected by the suction ring, which has been shown to reduce goblet cell density postsurgery $[50,51]$.

On the contrary, a former cataract surgery technique, extracapsular cataract extraction, requires a larger incision and is expected to induce more corneal sensitivity loss [52] and thus induce more signs and symptoms of dryness post-surgery [53]. Similarly, certain types of IOLs such as accommodative [54] designs require a larger incision for insertion. In this sense, reduced incisions lead to a faster corneal sensitivity recovery (within 1-3 months) compared to larger incisions, and only to a focal diminution of corneal sensation [44,42]. In the same way, micro incisional procedures such as phacoemulsification or the insertion of foldable IOLs are expected to induce less hypoesthesia than conventional techniques [43,44,52,55]. Additionally, incision shape, depth and regularity clearly impact post-surgery healing [42,44].

Finally, potential toxicity of antiseptic agents used during the surgical procedure as well as topical multi-dose eyedrops with preservatives seem to play a role in the onset of dry eye signs and symptoms. Benzalkonium chloride (BAK), is one of the most commonly used preservatives in ocular topical drugs and is recognized to induce, apart from goblet cells apoptosis, conjunctival squamous metaplasia, disruption of the corneal epithelium barrier and TF instability among 


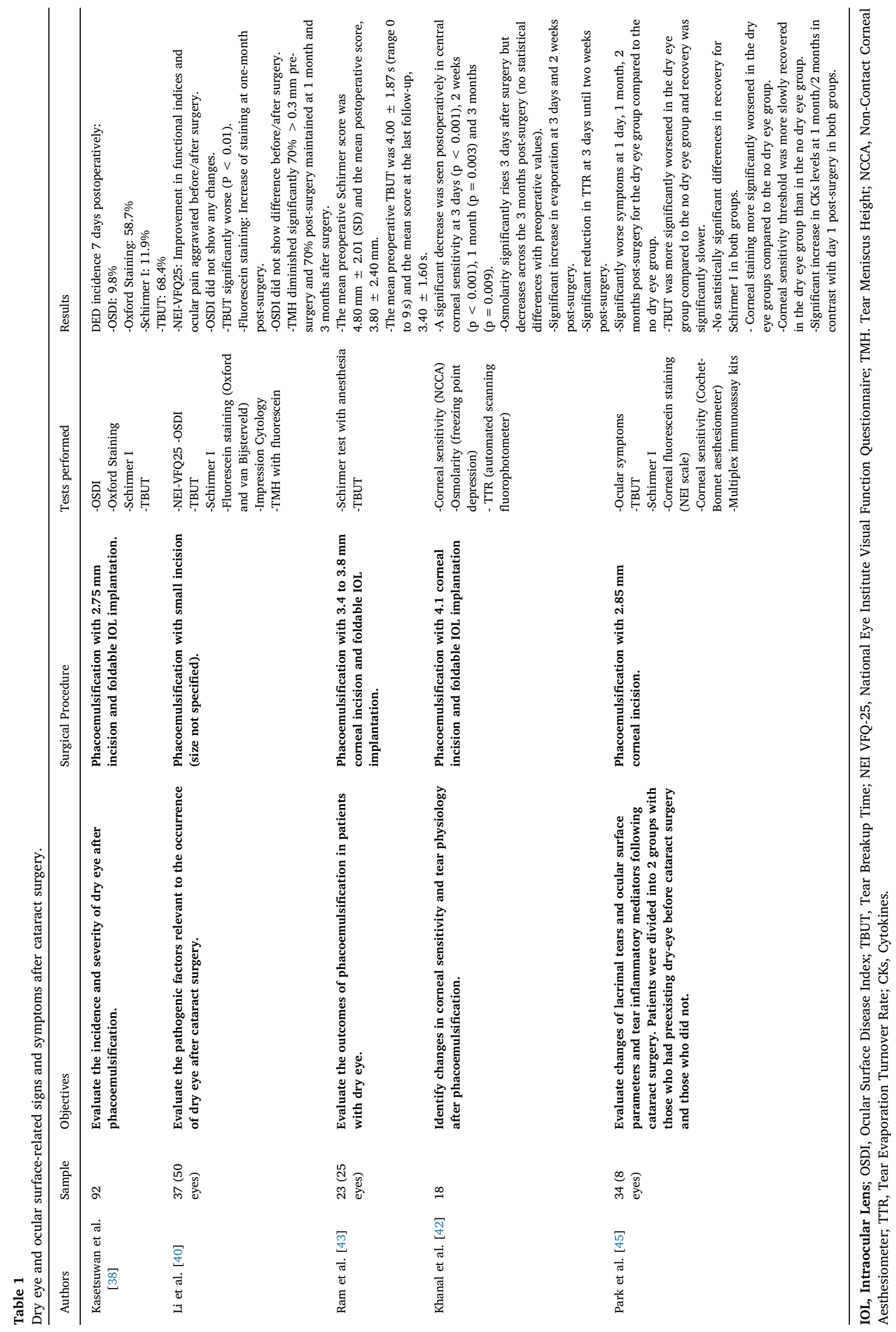


others [[35] [56],].

\subsection{Corneal refractive surgery}

\subsubsection{LASER IN-SITU KERATOMILEUSIS}

Laser in-situ keratomileusis (LASIK) is a surgical procedure in which a corneal flap (around 120-160 $\mu \mathrm{m}$ ) is created and then reclined (lifted) in order to proceed to the stromal ablation. Flap creation was initially performed using blades (microkeratome), but the emergence of newer technologies such as femtosecond lasers [48] are less invasive, reducing the signs of induced dry eye [34]. Once the flap is reclined, ablation is performed and destroys mid stromal nerves. Consequently, LASIK induces damage to the cornea during both the flap creation where the subbasal nerves are cut, and the excimer laser stromal ablation where stromal nerve trunks are destroyed by the laser [57]. Specifically, it is estimated that there is a $90 \%$ reduction of central nerve fiber density in the first month following surgery [58] and some studies report that corneal sensitivity does not return to baseline levels until 2-5 years post-surgery [59].

Consequently, DED is one, if not the most, common adverse effect of LASIK $[34,60,61]$. When performed on DED patients, the LASIK procedure worsens numerous tear metrics (tear volume [62], tear stability [63-,64,65], osmolarity [66,67]) and staining [63]. (Table 2). In parallel, ocular symptoms of dryness tend to reach a peak between one week and three months after surgery, regardless of preexistent dry eye [48,59,64,65,68,70-80].

LASIK monovision is a valuable option for the presbyopic population [81-83] and other new multifocal LASIK techniques, such as presbyLASIK, for which the excimer laser produces a multifocal corneal ablation profile, have also been developed [84]. Nonetheless, corneal monovision currently offers the highest 'success' rate (reaching $90 \%$ success) [85].

Shoja and Besharati, found a statistically significant effect of age on corneal sensitivity after LASIK [65]; patients developing dry eye after LASIK were significantly more likely to be older in comparison to patients who did not develop dry eye. Kanellopoulus also noted a significant association between age and clinically significant dry eye following LASIK [86]. Similarly, Price et al. in a multivariate model that controlled for dry symptoms at baseline, reported older age as one of the main factors associated with dry eye symptoms 3 years after LASIK [87]. On the contrary, many recent studies discard age as an important risk factor for post-LASIK tear dysfunction or dry eye. For example, Golas and Manche found no statistically significant effect of age on dry eye scores obtained in 51 patients after LASIK [88]. In addition, De Paiva et al. studied 35 adults, aged $24-54$ years, and found no association between older age and the risk for developing postoperative dry eye [69].

Given the major improvements in safety and efficacy of corneal refractive surgery in recent years, the demand for this type of procedure has considerably increased among the presbyopic population [83]. Albeit LASIK has shown to be successful in correcting refractive errors in presbyopic patients, studies evaluating outcomes of the surgery are still limited and present contradictory results. Nevertheless, the clinician must take into account that given the invasiveness of this technique, due to the flap creation, post-LASIK dry eye will remain a common complication. Given that preoperative tear function is thought to play an important role in long-term ocular surface integrity after LASIK [89], tear function should be assessed in detail for older patients considering this refractive surgery.

\subsubsection{Photorefractive keratectomy}

Photorefractive Keratectomy (PRK) is based on removal of the corneal epithelium using an alcohol solution following topical anesthesia (the corneal epithelium is discarded) [90]. The underlying corneal tissue is then reshaped using the excimer laser [more anterior in comparison to LASIK or Laser Assisted Subepithelial Keratectomy
(LASEK) procedures]. No flap is created for this procedure. Recovery takes longer than the LASIK technique, since it takes around a week for epithelial cells to regrow [90]. PRK induces a temporary decrease in subbasal corneal nerve density for up to a year, and complete recovery might take as long as two years [91]. In addition, studies report diminished tear secretion [92-94], tear stability [94,95], and corneal sensitivity $[96,97]$ in patients $3-6$ months post-surgery. (see Table 3 ).

As for LASIK, PRK may be performed as a presbyopia correction strategy by inducing monovision. In this regard, while part of the recent literature suggests no effects of age on patient-reported dry eye after PRK [98], other studies advise that the higher prevalence of DED along with corneal changes seen with advancing age may possibly hinder the healing process [99], affecting the final outcome of the surgery [100].

More studies regarding dry eye after corneal refractive surgery in late adulthood are required. Meanwhile, clinicians should pay particular attention to dry eye signs and symptoms before undertaking PRK in older age groups, as the deteriorating effect of the surgery on the ocular surface may worsen an already unbalanced ocular environment.

\subsubsection{Laser assisted subepithelial keratectomy}

The main difference between LASEK and PRK is that the peeled corneal epithelium, called an epithelial flap (which is discarded in the PRK technique), is repositioned after photoablation (the LASIK procedure uses a stromal flap [101]). Alcohol is used to weaken adhesions between the stroma and epithelium [102]. Factors such as alcohol concentration (usually between 18-25\%) and exposure time play a key role in postoperative healing [103]. Autrata et al. compared 184 eyes of 92 patients between PRK and LASEK with 2 years follow-up [104]. The authors concluded that LASEK provided significantly quicker recovery and reduced pain and haze level compared to conventional PRK. [104] (See Table 4).

Similar to LASIK and PRK, LASEK may be applied in older age groups to treat presbyopia using monovision. Increasing age can considerably influence LASEK postoperative outcomes. For example, age has shown to increase the prevalence of postoperative complications [108], reduce predictability [109] and increase healing time [110] after LASEK.

To date, no studies have evaluated the effects of LASEK surgery on $\mathrm{TF}$ in late adulthood. Based on the results of studies obtained from the general population, lower postoperative dry eye signs and symptoms compared to other corneal ablation techniques are also expected in prebyopes and elderly patients. Besides this, considering the afore mentioned, older age groups may be more susceptible to post-LASEK dry eye related complications.

\subsubsection{Small incision lenticule extraction}

The advent of lasers in the ophthalmic field to perform corneal refractive surgery has led to the concept of lenticule extraction. Recently, Small Incision Lenticule Extraction (SMILE) has been developed to perform corneal reshaping [111]. This refractive procedure uses a femtosecond laser to create a corneal lenticule that is extracted through a small incision [111].

SMILE no longer requires excimer laser ablation or the creation of a flap, making this technique less invasive than LASIK. The absence of a flap, reduces corneal inflammation and keratocyte damage ${ }^{112}$ and resulting in less iatrogenic dry eye [113], compared to other corneal refractive strategies, such as LASIK. Denoyer et al. found that $80 \%$ of SMILE patients did not use any eye drops 6 months post-surgery compared to $57 \%$ in the LASIK group, with $20 \%$ of the LASIK group requiring daily and frequent use of tear substitutes or even gels [58]. Higher tear osmolarity and lower TBUT, Schirmer score and corneal sensitivity were also observed in the LASIK group (see Table 5). Moreover, according to Li et al., SMILE patients reported less DED symptoms and had higher subbasal nerve density three months after surgery in comparison with LASIK patients [114].

SMILE monovision represents an additional corneal refractive 


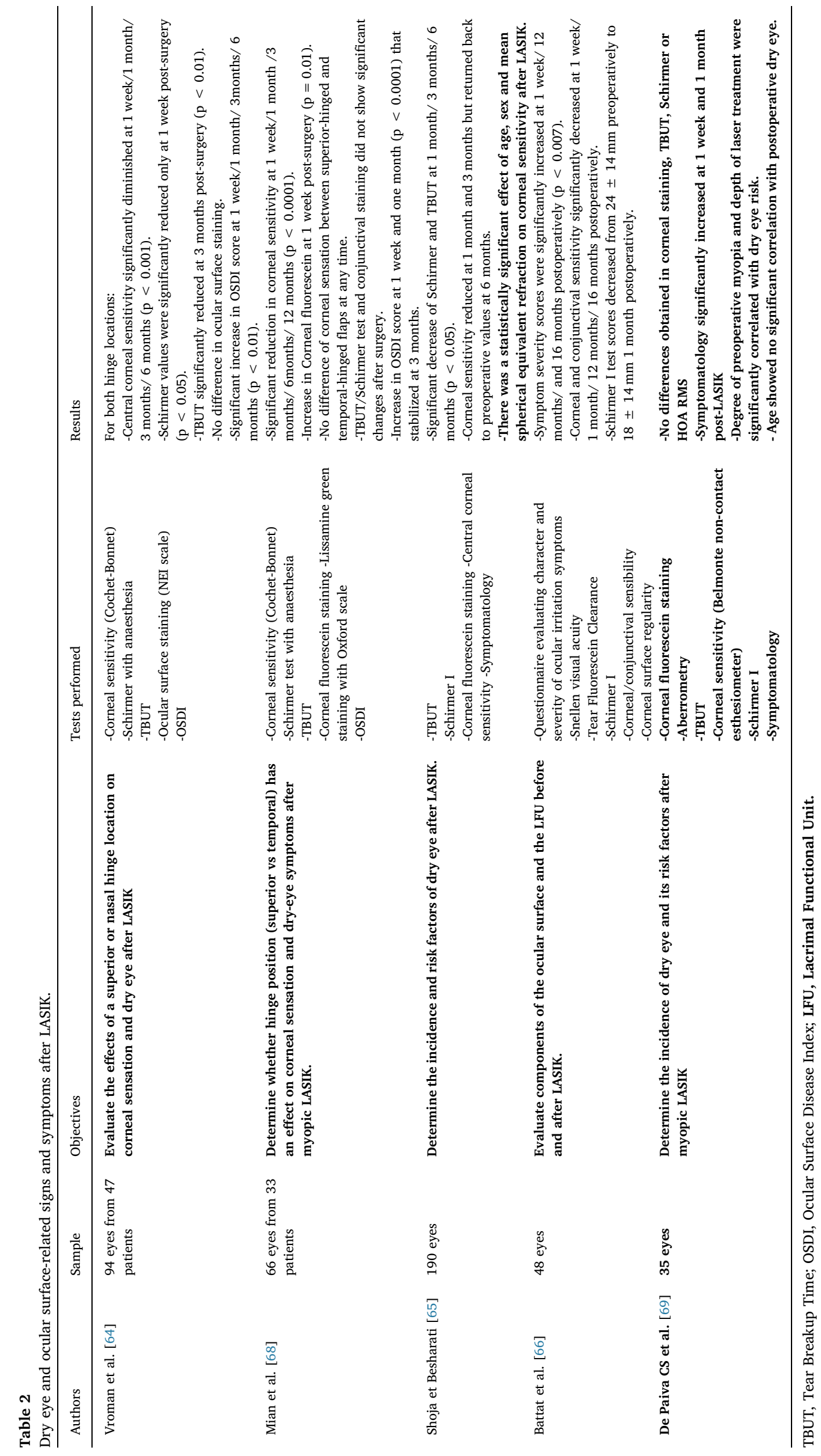


surgical technique for presbyopia correction. This technique has shown to be a safe and effective option, yielding predictable outcomes for treating patients with presbyopia [115]. While more studies based on late adulthood are needed, the advantages of this technique in relation to tear function, found in the general population, are also expected to benefit older individual's.

Importantly, older patients tend to have a greater stromal response to SMILE and more unpredictable refractive outcomes [118]. Older age has been identified as a risk factor for residual refractive error following SMILE that requires enhancement procedures (PRK) [116], speculated to result from wound healing and biomechanical characteristics in older corneas [117]. Consequently, as in the previous strategies, the clinician must consider the potentially increased effects of SMILE on the TF and ocular dryness with increasing age.

\subsubsection{Corneal onlays/inlays}

The main advantage of corneal onlays/inlays over the previously described techniques is that no tissue removal is needed [119]. Corneal onlays/inlays are optical devices designed to change corneal curvature or modify its optical properties, either by altering the refractive index to induce bifocal optics or by using small aperture optics in order to increase depth of focus [120]. Nowadays, femtosecond laser is widely used as it provides a more dependable flap than a microkeratome [121] and allows for the creation of stromal pockets, improving the accuracy of implantation depth and inlay centration [122].

Dry eye after corneal inlay implantation is mainly due to the flap creation which is basically the same technique as for LASIK surgery [123]. However, since no laser ablation is applied to the corneal stroma, less deep nerve damage is expected to occur in comparison with LASIK. In addition, the stromal pocket technique is less invasive than the flap technique and as such, a reduced incidence of dry eye postsurgery is expected as well as a shorter recovery period [124,125].

Tomita et al. examined the postoperative outcomes of 277 patients after LASIK and small-aperture corneal inlay implantation for hyperopic presbyopia [126]. The authors found no significant effect of age on the rates or severity of subjective symptoms, including dryness. Nevertheless, they underlined that taking age into account might help achieve optimum postoperative outcomes and improved patient satisfaction [126].

To conclude, the ocular surface should be carefully evaluated, and treated when required, before and after inlay implantation. As pre-existing dry eye is common in the presbyopic population it will likely be exacerbated by the creation of a pocket or a flap. Further studies are needed to assess the long-term outcomes of the lamellar cut and tunnel incision performed for the refractive inlay and small aperture optics implants [127] on dry eye signs and symptoms.

\section{Contact lenses}

Various CL options for presbyopic correction are available on the market: including single vision (combination of distance correction CLs and reading glasses), monovision, bifocal designs and multifocal designs [128]. However, not every CL wearer is able to achieve acceptable comfort and vision during CL wear and this can eventually lead to discontinuation and dropout; CL Discomfort (CLD) (24\%) and dryness (20\%) being the primary reasons of discontinuation [129-131]. In this regard, the TFOS International Workshop on CLD has extensively reviewed the problem of CLD and associated dryness [132]. According to recent findings, the mechanisms involved in CLD seem to share common pathways with DED [133-135], initiating a closed loop of inflammation as described by Baudouin et al. [136]

When a CL is fitted on a patient's eye, TF is disturbed leading to an increase in evaporation rate and dewetting [137] and possibly impacting the function of the MGs $[129,131,137]$. Specifically, DED in CL wearers is associated with a reduction in wearing time [138], increased risk of desiccation [122] (and raised osmolarity) [139] and thus higher 


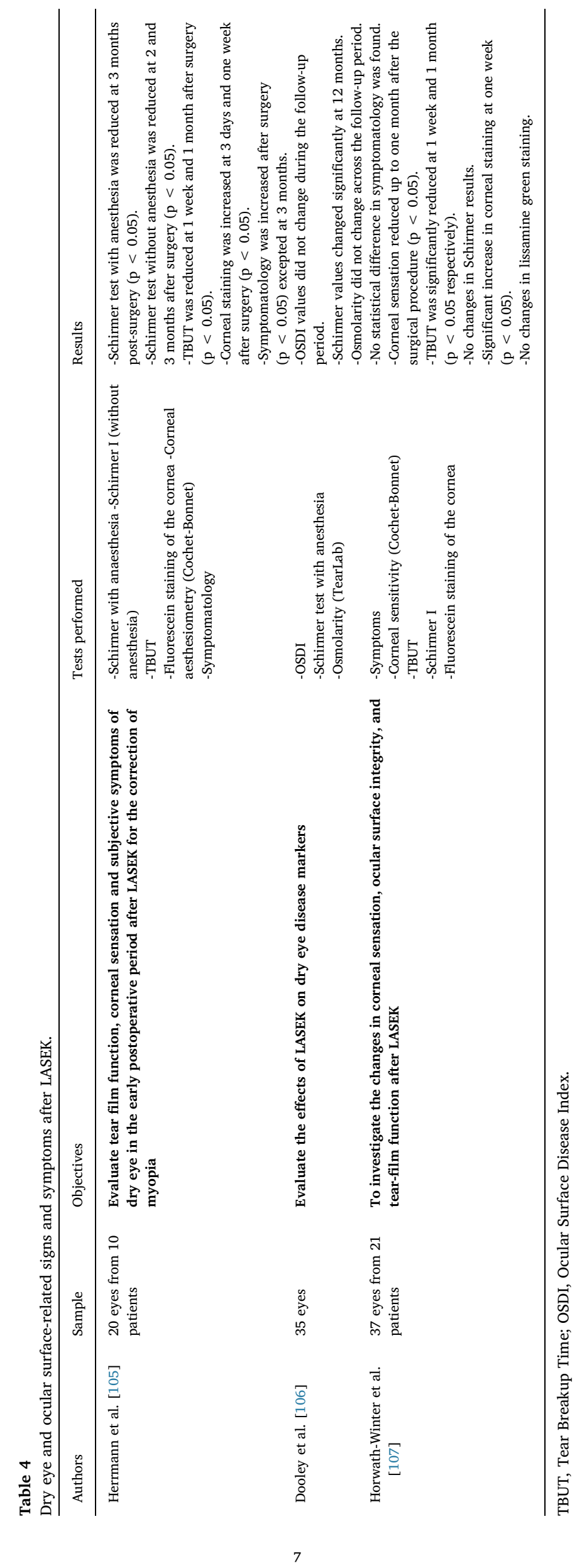




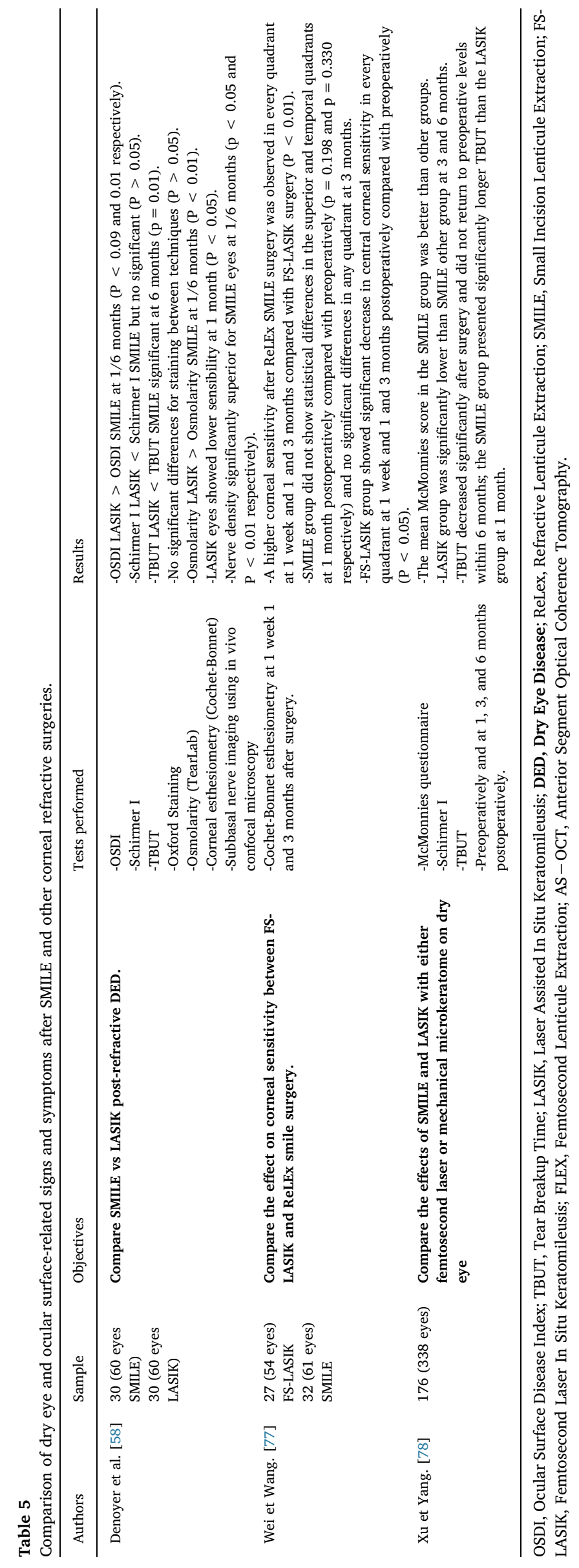


Table 6

Summary of the dry eye-related main outcomes up to date in an aging population after the different procedures addressed in this review.

\begin{tabular}{|c|c|}
\hline Procedure & Main Outcomes and Important Considerations \\
\hline Cataract surgery & $\begin{array}{l}\text { - Worsen tear film metrics }[34-36,38,40,43,44] \text {, reduce corneal sensitivity }[41,42,45] \text { and decrease goblet cell density [34-37] up to } 3 \text { months } \\
\text { post-surgery. } \\
\text { - Larger corneal incisions for lens insertion are expected to induce more nerve damage and thus, sign and symptoms of dryness post-surgery } \\
\text { [42,44]. } \\
\text { - FLACS offers a more accurate cutting edge, better safety and improved clinical outcomes [46-49]. However conjunctival pressure by the suction } \\
\text { ring reduces goblet cell density and contributes to postoperative DED [50,51]. } \\
\text { - Dry eye risk factors after the surgery are related to disruption of corneal nerves and harm to the epithelia through the surgical procedure [34-38]. } \\
\text { Incision shape, depth and regularity clearly impact post-surgery healing [42-44]. } \\
\text {-Toxicity of antiseptic agents used during the surgical procedure and topical multi-dose eyedrops with preservatives seem to play a role in the onset } \\
\text { of dry eye signs and symptoms }{ }^{35.56} \text {. }\end{array}$ \\
\hline LASIK & $\begin{array}{l}\text { - Successful in correcting refractive errors in presbyopic patients [81-83]. } \\
\text { - No consensus that older age impacts post-LASIK dry eye. } \\
\text { - The LASIK surgical process induces double damage to the cornea; during the flap creation and during the excimer laser stromal ablation, } \\
\text { increasing the probability of postoperative dry eye }[34,60,61] \text {. } \\
\text { - Detailed assessment of tear film and ocular surface should be carried out before performing this surgery in older age groups. } \\
\text {-Given the probability of post-LASIK dry eye, LASIK should only be applied in presbyopic patients with a good quality ocular surface and tear film. }\end{array}$ \\
\hline PRK & $\begin{array}{l}\text { - Recent studies report no effects of age on patient-reported dry eye after PRK [98]. } \\
\text { - Older age, however, may possibly hinder the healing process affecting the outcome of the surgery [99]. } \\
\text { - More studies regarding dry eye after PRK in the late adulthood are required. } \\
\text { - Particular attention should be taken in older age groups before undertaking PRK as the surgery may worsen an already unbalanced ocular surface } \\
\text { environment. }\end{array}$ \\
\hline LASEK & $\begin{array}{l}\text { - Age increases the prevalence of postoperative complications [108], reduces predictability [109] and increases healing time [110] after LASEK. } \\
\text { - To date no studies have evaluated the effects of LASEK surgery on tear film in late adulthood. } \\
\text { - Quicker recovery, reduced pain and less postoperative dry eye compared to other corneal refractive surgeries in the general population [104]. } \\
\text { - Lower postoperative dry eye signs and symptoms compared to other corneal ablation techniques are expected in the elderly as well. }\end{array}$ \\
\hline SMILE & $\begin{array}{l}\text { - A safe and effective option, yielding predictable outcomes for treating patients with presbyopia [115]. } \\
\text { - Reduced corneal inflammation and keratocyte damage [112] and less iatrogenic dry eye [113], compared to other corneal refractive strategies, } \\
\text { mainly due to the absence of a flap. } \\
\text {-Older age is thought to increase the risk of enhancement [116]. } \\
\text {-Older patients tend to have more stromal response and unpredictable outcomes [118]. }\end{array}$ \\
\hline CORNEAL ONLAYS/INLAYS & $\begin{array}{l}\text { - Dry eye symptoms after inlay implantation in presbyopes are mostly mild to moderate [124-126]. } \\
\text { - Less deep nerve damage is expected to occur in comparison with LASIK due to the absence of corneal ablation. } \\
\text { - Stromal pocket may offer reduced dry eye symptomatology in comparison to corneal flap }[124,125] \text {. } \\
\text {-No significant differences on symptom severity has been obtained between groups of different ages [126]. }\end{array}$ \\
\hline CLs & $\begin{array}{l}\text { - Age has shown to be the main factor influencing CL retention rate [145]. } \\
\text { - Presbyopic population might be more susceptible to dryness-related comfort problems, eventually leading to CLD and dropout [146]. } \\
\text { - Using a low rigidity CL on a daily disposable modality seems to be the most beneficial option for this group of patients [148,149]. } \\
\text { - SCLs can be a good optical platform for multifocality and a protection mechanism for the ocular surface, with reduced impact on the tear film } \\
\text { [150-153]. }\end{array}$ \\
\hline
\end{tabular}

FLACS, Femtosecond Laser Assisted Cataract Surgery; DED, Dry Eye Disease; LASIK, Laser in Situ Keratomileusis; PRK, Photorefractive Keratectomy; LASEK, Laser Assisted Subepithelial Keratectomy; SMILE, Small Incision Lenticule Extraction; CLs, Contact Lenses.

rates of infection [140]. Furthermore, CL water content has been associated with CL related dry eye. In this regard, it is thought that high water content CL alters the lipid layer structure of the TF, possibly due to the affinity of the polar components of the lipid layer to the CL surface, causing disruption of the prelens TF and thus increasing evaporation and/or dewetting [130,133,141]. Modifying the fit, changing the CL material and wearing schedule, or even prescribing eyedrops are the main solutions available to alleviate dryness signs [141,142].

In addition, discomfort symptoms related to asthenopic eye strain (burning, irritation, ocular dryness and tearing) have been noted to be closely related to symptoms of dry eye [143] and CLD [132]. Consequently, DED-like symptoms may be partially explained by suboptimally corrected refractive error or binocular vision disorders in many CL wearers, particularly in older patients without near vision correction [144].

Additionally, the physiological changes of advancing age on the ocular surface and TF might decrease the tolerance for CLs and increase the risks of complications [130]. In fact, age has shown to be the main factor influencing CL retention rate [145]. Patel et al. suggest that the presbyopic population might be more susceptible to dryness-related comfort problems [146], mainly because of decreased TF stability, eventually leading to CLD and dropout. However, du Toit et al. found no differences in the TF, ocular surfaces and symptoms between younger and older presbyopic patients, after 6 months of CL wear, except for a shorter TBUT in the older group [147]. The authors pointed out that the dry eye signs and ratings obtained were comparable with figures previously reported for all age groups of CL wearers. Hence, they reflected that presbyopes should not be excluded from consideration for CL fitting and that the usual patient care tenets apply. Overall, evidence suggests that using a low rigidity CL on a daily disposable modality, especially hydrogel daily disposable CLs, could be beneficial when fitting patients with presbyopia [148,149].

In addition, over the past decade there has been a resurgence of interest in scleral CLs (SCLs). SCLs are large-diameter rigid gas permeable CLs that vault the cornea and limbus and are supported by the sclera. These characteristics avoid direct mechanical stress to the cornea and enable the protection and continuous sealed hydration of the ocular surface [152]. Consequently, SCLs are considered a good therapeutic approach for the treatment of patients with moderate to severe dry eye [152-154]. In particular, small diameter SCLs, also known as corneo-scleral or mini-scleral lenses, have been reported especially suitable for this population [155].

As mentioned previously, fitting CLs in a presbyopic population is more challenging in comparison with a younger cohort. However, presbyopic patients could benefit from wearing SCLs; multifocal designs present great advantages such as excellent centration and stability along with better optical quality, compared to conventional multifocal CLs [153]. In this sense, SCLs present dual advantages for this population as they can provide a stable optical platform for correcting presbyopia and protect the ocular surface by vaulting the cornea, reducing their impact on the TF.

Moreover, SCLs are considered a suitable option for aiding patients 
with corneal ectasia, irregularity, and dry eye after PRK and LASIK surgery $[156,157]$. In this regard, postoperative optical complications following laser surgery have been observed, particularly procedures conducted in the 1990's, when the importance of sufficient residual bed thickness and exclusion of both form fruste and manifest keratoconus were perhaps not appreciated [158]. Thirty years later many of these patients are now presbyopic and may benefit from treatment with SCL's.

\section{Conclusions}

Aging processes challenge the ocular surface directly by inducing drastic changes to the LFU. Additionally, ocular surface integrity can be jeopardized through surgical interventions involving the cornea and CL fitting, potentially initiating a closed loop of inflammation leading to DED (Table 6). Given that preoperative tear function is thought to play an important role in long-term ocular surface integrity after surgical procedures, the clinician must consider the potentially greater adverse effects of surgery on the TF and ocular dryness with increasing age. More than in any other age group, postoperative ocular dryness is highly dependent on the invasiveness of the surgical technique, mostly related to corneal nerve damage. Similarly, CLD and dryness CL wearing presbyopes may be influenced to some extent by the lens material and wearing schedule. Newer CL designs including SCLs may be particularly useful for presbyopes with DED since they provide a stable optical platform and protection and constant hydration of the ocular surface. Further studies are still needed to assess long-term outcomes of recent advances in refractive surgeries and CL designs on dry eye signs and symptoms in older adults.

\section{Declaration of Competing Interest}

The authors have no conflicts of interest to disclose.

\section{Acknowledgements and Disclosure}

The authors have no proprietary interest in any of the materials mentioned in this article. This project has received funding from the European Union's Horizon 2020 research and innovation programme under the Marie Sklodowska-Curie grant agreement No. 642760 EDEN ITN-EJD Project Horizon 2020.

\section{References}

[1] M.E. Stern, R.W. Beuerman, R.I. Fox, et al., The Pathology of Dry Eye: the interaction between the ocular surface and lacrimal glands, Cornea 17 (1998) 584-589.

[2] D.A. Sullivan, Lacrimal gland, tear film and dry eye syndrome: basic science and clinical relevance, Springer, NYC, 1994.

[3] J.P. Craig, K.K. Nichols, E.K. Akpek, et al., TFOS DEWS II definition and classification report, Ocul Surf 15 (2017) 276-283.

[4] E.M. Rocha, M. Alves, J.D. Rios, et al., The aging lacrimal gland: changes in structure and function, Ocul Surf 6 (2008) 162-174.

[5] A.B. El-Fadaly, E.A. El-Shaarawy, A.A. Rizk, et al., Age-related alterations in the lacrimal gland of adult albino rat: a light and electron microscopic study, Ann Anat 196 (2014) 336-351.

[6] H. Obata, S. Yamamoto, H. Horiuchi, et al., Histopathologic study of human lacrimal gland. Statistical analysis with special reference to aging, Ophthalmology 102 (1995) 678-686.

[7] P. Chhadva, A.L. McClellan, C.R. Alabiad, et al., Impact of eyelid laxity on symptoms and signs of dry eye disease, Cornea 35 (2016) 531-535.

[8] Y.A. Alghamdi, C. Mercado, A.L. McClellan, et al., Epidemiology of meibomian gland dysfunction in an elderly population, Cornea 35 (2016) 731-735.

[9] H. Pult, B.H. Riede-Pult, Impact of conjunctival folds on central tear meniscus height, Invest Ophthalmol Vis Sci 56 (2015) 1459-1466.

[10] K. Gumus, S.C. Pflugfelder, Increasing prevalence and severity of conjunctivochalasis with aging detected by anterior segment optical coherence tomography, Am J Ophthalmol 155 (2013) 238-242.

[11] W. Chao, C. Belmonte, J.M.B.D. Castillo, et al., Report of the inaugural meeting of the TFOS $\mathrm{i}(2)=$ initiating innovation series: targeting the unmet need for dry eye treatment, Ocul Surf 14 (2016) 264-316.

[12] O.D. Schein, B. Munoz, J.M. Tielsch, et al., Prevalence of dry eye among the elderly, Am J Ophthalmol 124 (1997) 723-728.
[13] S.E. Moss, R. Klein, B.E. Klein, Long-term incidence of dry eye in an older population, Optom Vis Sci 85 (2008) 668-674.

[14] The Lancet. Series on Ageing. November (6), 2014. Available at: http://www, thelancet.com/series/ageing. (Accessed 2 October 2018).

[15] F. Stapleton, M. Alves, V.Y. Bunya, et al., TFOS DEWS II epidemiology report, Ocul Surf 15 (2017) 334-365.

[16] P.A. Papadopoulos, A.P. Papadopoulos, Current management of presbyopia, Middle East Afr J Ophthalmol 21 (2014) 10-17.

[17] I. Patel, S.K. West, Presbyopia: prevalence, impact, and interventions, Community Eye Health 20 (2007) 40-41.

[18] American Optometric Association, Care of the patient with presbyopia, American Optometric Association, St. Louis, 2010.

[19] K.D. Frick, S.M. Joy, D.A. Wilson, et al., The global burden of potential productivity loss from uncorrected presbyopia, Ophthalmology 122 (2015) 1706-1710.

[20] W.N. Charman, Developments in the correction of presbyopia I: spectacle and contact lenses, Ophthalmic Physiol Opt 34 (2014) 8-29.

[21] W. He, D. Goodkind, P.R. Kowal, An aging world: 2015, United States Census Bureau, 2016.

[22] B. Thylefors, A.D. Negrel, R. Pararajasegaram, et al., Global data on blindness, Bull World Health Organ 73 (1995) 115-121.

[23] P.A. Asbell, I. Dualan, J. Mindel, et al., Age-related cataract, Lancet 365 (2005) 599-609.

[24] N. Pescosolido, A. Barbato, R. Giannotti, et al., Age-related changes in the kinetics of human lenses: prevention of the cataract, Int J Ophthalmol 9 (2016) $1506-1517$.

[25] L.J. Muller, L. Pels, G.F. Vrensen, Ultrastructural organization of human corneal nerves, Invest Ophthalmol Vis Sci 37 (1996) 476-488.

[26] C. Belmonte, J.J. Nichols, S.M. Cox, et al., TFOS DEWS II pain and sensation report, Ocul Surf 15 (2017) 404-437.

[27] C.F. Marfurt, J. Cox, S. Deek, et al., Anatomy of the human corneal innervation, Exp Eye Res 90 (2010) 478-492.

[28] C. Belmonte, M.C. Acosta, J. Gallar, Neural basis of sensation in intact and injured corneas, Exp Eye Res 78 (2004) 513-525.

[29] S. Trikha, A.M. Turnbull, R.J. Morris, et al., The journey to femtosecond laserassisted cataract surgery: new beginnings or false dawn? Eye 27 (2013) 461-473.

[30] D.S. Grewal, T. Schultz, S. Basti, et al., Femtosecond laser-assisted cataract surgery-current status and future directions, Surv Ophthalmol 61 (2016) 103-131.

[31] G. Brian, H. Taylor, Cataract blindness - challenges for the 21st century, Bull World Health Org 79 (2001) 249-256.

[32] R. Montés-Micó, Role of the tear film in the optical quality of the human eye, J Cataract Ref Surg 33 (2007) 1631-1635.

[33] R. Montés-Micó, J. Alió, N. Charman, Dynamic changes in the tear film in dry eyes, Invest Ophtalmol Vis Sci 46 (2005) 1615-1619.

[34] M.Q. Salomão, R. Ambrósio, S.E. Wilson, Dry eye associated with laser in situ keratomileusis: mechanical microkeratome versus femtosecond laser, J Cataract Refract Surg 35 (2009) 1756-1760.

[35] T.D. Walker, Benzalkonium toxicity, Clin Exp Ophthalmol 32 (2004) 657.

[36] Y.K. Cho, M.S. Kim, Dry eye after cataract surgery and associated intraoperative risk factors, Korean J Ophthalmol 23 (2009) 65-73.

[37] K. Nakamori, M. Odawara, T. Nakajima, et al., Blinking is controlled primarily by ocular surface conditions, Am J Ophthalmol 124 (1997) 24-30.

[38] N. Kasetsuwan, V. Satitpitakul, T. Changul, et al., Incidence and pattern of dry eye after cataract surgery, PLoS One 8 (2013) e78657.

[39] M. Calonge, A. Enriquez-de-Salamanca, Y. Diebold, et al., Dry eye disease as an inflammatory disorder, Ocul Immunol Inflamm 18 (2010) 244-253.

[40] X.-M. Li, L. Hu, J. Hu, et al., Investigation of Dry Eye Disease and analysis of the pathogenic factors in patients after cataract surgery, Cornea 26 (2007) 16-20.

[41] M. Kohlhaas, O. Stahlhut, J. Tholuck, et al., Development of corneal sensitivity after phacoemulsification with scleral tunnel incision, Klin Monbl Augenheilkd 211 (1997) 32-36.

[42] S. Khanal, A. Tomlinson, L. Esakowitz, et al., Changes in corneal sensitivity and tear physiology after phacoemulsification, Ophthalmic Physiol Opt 28 (2008) $127-134$.

[43] J. Ram, A. Gupta, G. Brar, et al., Outcomes of phacoemulsification in patients with dry eye, J Cataract Refract Surg 28 (2002) 1386-1389.

[44] T. Oh, Y. Jung, D. Chang, et al., Changes in the tear film and ocular surface after cataract surgery, Jpn J Ophthalmol 2 (2012) 113-118.

[45] Y. Park, H.B. Hwang, H.S. Kim, Observation of influence of cataract surgery on the ocular surface, PLoS One 11 (2016) e0152460.

[46] K.E. Donaldson, R. Braga-Mele, F. Cabot, et al., Femtosecond laser-assisted cat aract surgery, J Cataract Refract Surg 39 (2013) 1753-1763.

[47] Z. Nagy, A. Takacs, T. Filkorn, et al., Initial clinical evaluation of an intraocular femtosecond laser in cataract surgery, J Refract Surg 25 (2009) 1053-1060.

[48] A. Sugar, Ultrafast (femtosecond) laser refractive surgery, Curr Opin Ophthalmol 13 (2002) 246-249.

[49] D.V. Palanker, M.S. Blumenkranz, D. Andersen, et al., Femtosecond laser-assisted cataract surgery with integrated optical coherence tomography, Sci Transl Med 2 (2010) 58-85.

[50] N.J. Friedman, D.V. Palanker, G. Schuele, et al., Femtosecond laser capsulotomy, J Cataract Refract Surg 37 (2011) 1189-1198.

[51] J.L. Rodriguez-Prats, I.M. Hamdi, A.E. Rodriguez, et al., Effect of suction ring application during LASIK on goblet cell density, J Refract Surg 23 (2007) 559-562.

[52] R.S. Hoffman, I.H. Fine, M. Packer, New phacoemulsification technology, Curr Opin Ophthalmol 16 (2005) 38-43.

[53] R. Sitompul, G.S. Sancoyo, J.A. Hutauruk, et al., Sensitivity change in Cornea and 
tear layer due to incision difference on cataract surgery with either manual smallincision cataract surgery or phacoemulsification, Cornea 27 (2008) 13-18.

[54] A. Aristeidou, E.V. Taniguchi, M. Tsatsos, et al., The evolution of corneal and refractive surgery with the femtosecond laser, Eye Vis 14 (2015) 2-12.

[55] A. Kissner, M. Kohlhaas, E. Spörl, et al., Corneal aberrations before and after corneal and corneoscleral small incision cataract surgery, Klin Monbl Augenheilkd 224 (2007) 95-100.

[56] C. Baudouin, A. Labbé, H. Liang, et al., Preservatives in eyedrops: the good, the bad and the ugly, Prog Ret Eye Res 29 (2010) 312-334.

[57] M.P. Calvillo, J.W. Mclaren, D.O. Hodge, et al., Corneal reinnervation after LASIK: prospective 3-Year longitudinal study, Invest Opthalmol Vis Sci 45 (2004) 3991-3996.

[58] A. Denoyer, E. Landman, L. Trinh, Dry eye disease after refractive surgery: comparative outcomes of small incision lenticule extraction versus LASIK, Ophthalmology 122 (2015) 669-676.

[59] S.V. Patel, J.W. McLaren, K.M. Kittleson, et al., Subbasal nerve density and corneal sensitivity after laser in situ keratomileusis: femtosecond laser vs mechanical microkeratome, Arch Ophtalmol 128 (2010) 1413-1419.

[60] C. Chao, B. Golebiowski, F. Stapleton, The role of corneal innervation in LASIKinduced neuropathic dry eye, Ocul Surf 12 (2014) 32-45.

[61] R.T. Ang, D.A. Dartt, K. Tsubota, Dry eye after refractive surgery, Curr Opin Ophthalmol 12 (2001) 318-322.

[62] A. Tao, M. Shen, J. Wang, et al., Upper and lower tear menisci after laser in situ keratomileusis, Eye Contact Lens 36 (2010) 81-85.

[63] I. Toda, N. Asano-Kato, Y. Hori-Komai, et al., Laser-assisted in situ keratomileusis for patients with dry eye, Arch Ophthalmol 120 (2002) 1024-1028.

[64] D.T.1 Vroman, H.P. Sandoval, L.E. Fernández de Castro, et al., Effect of hinge location on corneal sensation and dry eye after laser in situ keratomileusis for myopia, J Cataract Refract Surg 31 (2005) 1881-1887.

[65] M. Shoja, M. Besharati, Dry eye after lasik for myopia: incidence and risk factors, Eur J Ophthalmol 17 (2007) 1-6.

[66] L. Battat, A. Macri, D. Dursun, et al., Effects of laser in situ keratomileusis on tear production, clearance, and the ocular surface, Ophthalmology 108 (2001) $1230-1235$.

[67] C. Chao, F. Stapleton, X. Zhou, et al., Structural and functional changes in corneal innervation after laser in situ keratomileusis and their relationship with dry eye, Graefes Arch Clin Exp Ophthalmol 253 (2015) 2029-2039.

[68] S.I. Mian, A.Y. Li, S. Dutta, et al., Dry eyes and corneal sensation after laser in situ keratomileusis with femtosecond laser flap creation, J Cataract Refract Surg 35 (2009) 2092-2098.

[69] C.S. De Paiva, Z. Chen, D.D. Koch, et al, The incidence and risk factors for developing dry eye after myopic LASIK, Am J Ophthalmol 141 (2006) 438-445.

[70] S.C. Pflugfelder, R.M. Corrales, C.S.D. Paiva, T helper cytokines in dry eye disease, Exp Eye Res 117 (2013) 118-125.

[71] J.C. Erie, J.W. McLaren, D.O. Hodge, et al., Recovery of corneal subbasal nerve density after PRK and LASIK, Am J Ophthalmol 140 (2005) 1059-1064.

[72] R. Ambrósio, T. Tervo, S.E. Wilson, LASIK-associated dry eye and neurotrophic epitheliopathy: pathophysiology and strategies for prevention and treatment, J Refract Surg 24 (2008) 396-407.

[73] B.V. Jagow, T. Kohnen, Corneal architecture of femtosecond laser and microkeratome flaps imaged by anterior segment optical coherence tomography, J Cataract Refract Surg 35 (2009) 35-41.

[74] D.B. Tran, M.A. Sarayba, Z. Bor, et al., Randomized prospective clinical study comparing induced aberrations with IntraLase and Hansatome flap creation in fellow eyes, J Cataract Refract Surg 31 (2005) 97-105.

[75] D.S. Durrie, G.M. Kezirian, Femtosecond laser versus mechanical keratome flaps in wavefront-guided laser in situ keratomileusis, J Cataract Refract Surg 31 (2005) $120-126$.

[76] T. Tervo, A. Vannas, K. Tervo, et al., Histochemical evidence of limited reinnervation of human corneal grafts, Acta Ophthalmol (Copenh) 63 (1985) 207-214.

[77] S. Wei, Y. Wang, Comparison of corneal sensitivity between FS-LASIK and femtosecond lenticule extraction (ReLEx flex) or small-incision lenticule extraction (ReLEx smile) for myopic eyes, Graefes Arch Clin Exp Ophthalmol 25 (2013) $1645-1654$.

[78] Y. Xu, Y. Yang, Dry Eye After Small Incision Lenticule Extraction and LASIK for Myopia, J Refract Surg 30 (2014) 186-190.

[79] S.I. Mian, R.M. Shtein, A. Nelson, et al., Effect of hinge position on corneal sensation and dry eye after laser in situ keratomileusis using a femtosecond laser, $\mathrm{J}$ Cataract Refract Surg 33 (2007) 1190-1194.

[80] Z. Liu, Y. Li, Z. Cheng, et al., Seven-year follow-up of LASIK for moderate to severe myopia, J Refract Surg 24 (2008) 935-940.

[81] S. Jain, R. Ou, D.T. Azar, Monovision outcomes in presbyopic individuals after refractive surgery, Ophthalmology 108 (2001) 1430-1433.

[82] D. Miranda, R.R. Krueger, Monovision laser in situ keratomileusis for pre-presbyopic and presbyopic patients, J Refract Surg 20 (2004) 325-328.

[83] P. López-Montemayor, J.E. Valdez-García, D. Loya-García, et al., Safety, efficacy and refractive outcomes of LASIK surgery in patients aged 65 or older, Int Ophthalmol 38 (2018) 1515-1520.

[84] J.S. Wolffsohn, L.N. Davies, Presbyopia: effectiveness of correction strategies, Prog Retin Eye Res 68 (2019) 124-143.

[85] E. Levinger, O. Trivizki, R. Pokroy, et al., Monovision surgery in myopic presbyopes: visual function and satisfaction, Optom Vis Sci 90 (2013) 1092-1097.

[86] A.J. Kanellopoulus, Incidence and management of symptomatic dry eye related to LASIK for myopia, with topical cyclosporine A, Clin Ophthalmol 13 (2019) 545-552.
[87] M.O. Price, D.A. Price, F.A. Bucci Jr.et al., Three-year longitudinal survey comparing visual satisfaction with LASIK and contact lenses, Ophthalmology 123 (2016) 1659-1666.

[88] L. Golas, E.E. Manche, Dry eye after laser in situ keratomileusis with femtosecond laser and mechanical keratome, J Cataract Refract Surg 37 (2011) 1476-1480.

[89] K. Konomi, L.L. Chen, R.S. Tarko, et al., Preoperative characteristics and a potential mechanism of chronic dry eye after LASIK, Invest Ophthalmol Vis Sci 49 (2008) 168-174.

[90] A.C. Browning, S. Shah, H.S. Dua, et al., Alcohol debridement of the corneal epithelium in PRK and LASEK: an electron microscopic study, Invest Ophthalmol Vis Sci 44 (2003) 510-513.

[91] J.C. Erie, Corneal wound healing after photorefractive keratectomy: a 3-year confocal microscopy study, Trans Am Ophthalmol Soc 101 (2003) 293-333.

[92] J.B. Lee, C.H. Ryu, J. Kim, et al., Comparison of tear secretion and tear film in stability after photorefractive keratectomy and laser in situ keratomileusis, J Cataract Refract Surg 26 (2000) 1326-1331.

[93] R. Nejima, K. Miyata, T. Tanabe, et al., Corneal barrier function, tear film stability, and corneal sensation after photorefractive keratectomy and laser in situ keratomileusis, Am J Ophthalmol 139 (2005) 64-71.

[94] A. Ozdamar, C. Aras, N. Karakas, et al., Changes in tear flow and tear film stability after photorefractive keratectomy, Cornea 18 (1999) 437-439.

[95] J.W. Hong, H.M. Kim, The changes of tear break up time after myopic excimer laser photorefractive keratectomy, Korean J Ophthalmol 11 (1997) 89-93.

[96] T. Ishikawa, S.B. Park, C. Cox, et al., Corneal sensation following excimer laser photorefractive keratectomy in humans, J Refract Corneal Surg (10) (1994) 417-422.

[97] J.J. Perez-Santonja, H.F. Sakla, C. Cardona, et al., Corneal sensitivity after photorefractive keratectomy and laser in situ keratomileusis for low myopia, Am J Ophthalmol 127 (1999) 497-504.

[98] Y. Murakami, E.E. Manche, Prospective, randomized comparison of self-reported postoperative dry eye and visual fluctuation in LASIK and Photorefractive Keratectomy, Ophthalmology 119 (2012) 2220-2224.

[99] I.K. Gipson, Age-related changes and diseases of the ocular surface and cornea, Invest Ophthalmol Vis Sci 54 (2013) ORSF48- ORSF53.

[100] P.S. Hersh, O.D. Schein, R. Steinert, Characteristics influencing outcomes of excimer laser photorefractive keratectomy. Summit Photorefractive Keratectomy Phase III Study Group, Ophthalmology 103 (1996) 1962-1969.

[101] R. Ambrósio, S. Wilson, LASIK vs LASEK vs PRK: advantages and indications, Semin Ophthalmol 18 (2003) 2-10.

[102] S. Shah, S.J. Doyle, A. Chatterjee, et al., Comparison of $18 \%$ ethanol and mechanical debridement for epithelial removal before photorefractive keratectomy, $\mathrm{J}$ Refract Surg 14 (Suppl. 2) (1998) S212-214.

[103] S.Y. Kim, W.J. Sah, Y.W. Lim, et al., Twenty percent alcohol toxicity on rabbit corneal epithelial cells: electron microscopic study, Cornea 21 (2002) 388-392.

[104] R. Autrata, J. Rehurek, Laser-assisted subepithelial keratectomy for myopia: twoyear follow-up, J Cataract Refract Surg 29 (2003) 661-668.

[105] W.A. Herrmann, C.P. Shah, C.W.V. Mohrenfels, et al., Tear film function and corneal sensation in the early postoperative period after LASEK for the correction of myopia, Graefes Arch Clin Exp Ophthalmol 243 (2005) 911-916.

[106] I. Dooley, F. Darcy, M. Okeefe, Comparison of dry-eye disease severity after lase in situ keratomileusis and laser-assisted subepithelial keratectomy, J Cataract Refract Surg 38 (2012) 1058-1064.

[107] J. Horwath-Winter, B. Vidic, G. Schwantzer, et al., Early changes in corneal sensation, ocular surface integrity, and tear-film function after laser-assisted subepithelial keratectomy, J Cataract Refract Surg 30 (2004) 2316-2321.

[108] J. Galindo, A. Fadlallah, S. Robinson, et al., Risk factors for loss of epithelial flap integrity in laser-assisted subepithelial keratectomy surgery, J Cataract Refract Surg 42 (2016) 591-595.

[109] M. Garcia-Gonzalez, J. Gros-Otero, I. Rodrifuez-Perez, et al., Effect of age on visual and refractive results after laser-assisted subepithelial keratomileusis (LASEK) with adjuvant use of mitomycin C, J Optom 12 (2019) 92-98.

[110] J.H. Rhim, J.H. Kim, E.J. Yeo, et al., Caveolin-1 as a novel indicator of woundhealing capacity in aged human corneal epithelium, Mol Med 16 (2010) 527-534.

[111] D.Z. Reinstein, T.J. Archer, M. Gobbe, Small incision lenticule extraction (SMILE) history, fundamentals of a new refractive surgery technique and clinical outcomes, Eye Vis (Lond) 1 (2014) 1.

[112] Z. Dong, X. Zhou, J. Wu, et al., Small incision lenticule extraction (SMILE) and femtosecond laser LASIK: comparison of corneal wound healing and inflammation, Br J Ophthalmol 98 (2014) 263-269.

[113] M. Moshirfar, M.V. McCaughey, D.Z. Reinstein, et al., Small-incision lenticule extraction, J Cataract Refract Surg 41 (2015) 652-665.

[114] M. Li, L. Niu, B. Qin, et al., Confocal comparison of corneal reinnervation after small incision lenticule extraction (SMILE) and femtosecond laser in situ keratomileusis (FS-LASIK), PLoS One 8 (2013) e81435.

[115] N. Luft, J. Siedlecki, W. Sekundo, et al., Small incision lenticule extraction (SMILE) monovision for presbyopia correction, Eur J Ophthalmol 28 (2018) 287-293.

[116] Y.C. Liu, M. Rosman, J.S. Mehta, Enhancement after small-incision lenticule extraction: incidence, risk factors, and outcomes, Ophthalmology 124 (2017) 813-821.

[117] J.Ø Hjortdal, A.H. Vestergaard, A. Ivarsen, et al., Predictors for the outcome of small incision lenticule extraction for myopia, J Refract Surg 28 (2012) 865-871.

[118] M.V. Netto, R.R. Mohan, R. Ambrósio Jr.et al., Wound healing in the cornea: a review of refractive surgery complications and new prospects for therapy, Cornea 24 (2005) 509-522.

[119] R.L. Lindstrom, S.M. Macrae, J.S. Pepose, et al., Corneal inlays for presbyopia correction, Curr Opin Ophthalmol 24 (2013) 281-287. 
[120] E.M. Arlt, E.M. Krall, S. Moussa, et al., Implantable inlay devices for presbyopia: the evidence to date, Clin Ophthalmol 9 (2015) 129-137.

[121] T.P. Callou, R. Garcia, A. Mukai, et al., Advances in femtosecond laser technology, Clin Ophthalmol 10 (2016) 697-703.

[122] G.O. Waring, S.D. Klyce, Corneal inlays for the treatment of presbyopia, Int Ophthalmol Clin 51 (2011) 51-62.

[123] ÖF. Yilmaz, N. Alagöz, G. Pekel, et al., Intracorneal inlay to correct presbyopia: long-term results, J Cataract Refract Surg 37 (2011) 1275-1281.

[124] P. Binder, New femtosecond laser software technology to create intrastromal pockets for corneal inlays, ARVO 51 (2010) 2868.

[125] A.N. Limnopoulou, D.I. Bouzoukis, G.D. Kymionis, et al., Visual outcomes and safety of a refractive corneal inlay for presbyopia using femtosecond laser, J Refract Surg 29 (2013) 12-19.

[126] M. Tomita, Waring GO $4^{\text {th }}$. One-year results of simultaneous laser in situ keratomileusis and small-aperture corneal inlay implantation for hyperopic presbyopia: comparison by age, J Cataract Refract Surg 41 (2015) 152-161.

[127] A.K. Dexl, J. Ruckhofer, W. Riha, et al., Central and peripheral corneal iron deposits after implantation of a smallaperture corneal inlay for correction of presbyopia, J Refract Surg 27 (2011) 876-880.

[128] E.S. Bennett, Contact lens correction of presbyopia, Clin Exp Optom 91 (2008) 265-278.

[129] K. Richdale, L.T. Sinnott, E. Skadahl, et al., Frequency of and factors associated with contact Lens dissatisfaction and discontinuation, Cornea 26 (2007) 168-174.

[130] K. Dumbleton, C.A. Woods, L.W. Jones, et al., The impact of contemporary contact lenses on contact Lens discontinuation, Eye Contact Lens 39 (2013) 92-98.

[131] G. Young, J. Veys, N. Pritchard, et al., A multi-centre study of lapsed contact lens wearers, Ophthalmic Physiol Opt 22 (2002) 516-527.

[132] J.J. Nichols, M.D. Willcox, A.J. Bron, et al., The TFOS international workshop on contact Lens discomfort: executive summary, Invest Ophthalmol Vis Sci 54 (2013) TFOS7-TFOS13.

[133] A.S. Dogan, C. Gurdal, N. Arslan, Corneal confocal microscopy and dry eye findings in contact lens discomfort patients, Cont Lens Anterior Eye 41 (2018) 101-104.

[134] H. Wagner, R.L. Chalmers, G.L. Mitchell, et al., Risk factors for interruption to soft contact lens wear in children and young adults, Optom Vis Sci 88 (2011) 973-980.

[135] D.M. Robertson, The effects of silicone hydrogel lens wear on the corneal epithelium and risk for microbial keratitis, Eye Contact Lens 39 (2013) 67-72.

[136] C. Baudouin, P. Aragona, E. Messmer, et al., Role of hyperosmolarity in the pathogenesis and management of dry eye disease: proceedings of the OCEAN group meeting, Ocul Surf 11 (2013) 246-258.

[137] K. Dumbleton, B. Caffery, M. Dogru, et al., The TFOS international workshop on contact Lens discomfort: report of the subcommittee on epidemiology, Invest Ophthalmol Vis Sci 54 (2013) TFOS20-36.

[138] T. Harknett, T. Bowden, H. Shadbolt, et al., Five years after dispensing — are contact lenses a success? Cont Lens Anterior Eye 24 (2001) 127-128.

[139] J.J. Nichols, L.T. Sinnott, Tear film, contact lens, and patient-related factors associated with contact lens-related dry eye, Invest Ophthalmol Vis Sci 47 (2006)
1319-1328.

[140] G.M. Bruinsma, H.C. van der Mei, H.J. Busscher, Bacterial adhesion to surface hydrophilic and hydrophobic contact lenses, Biomaterials 22 (2001) 3217-3224.

[141] D. Fonn, P. Situ, T. Simpson, Hydrogel lens dehydration and subjective comfort and dryness rating in symptomatic and asymptomatic contact lens wearers, Optom Vis Sci 76 (1999) 700-704.

[142] N. Efron, Contact lens wear is intrinsically inflammatory, Clin Exp Optom 100 (2016) 3-19.

[143] J.E. Sheedy, J.N. Hayes, J. Engle, Is all asthenopia the same? Optom Vis Sci 80 (2003) 732-739.

[144] A.L. Sheppard, J.S. Wolffsohn, Digital eye strain: prevalence, measurement and amelioration, BMJ Open Ophthalmol 3 (2018) e000146.

[145] A. Sulley, G. Young, C. Hunt, Factors in the success of new contact lens wearers, Cont Lens Anterior Eye 40 (2017) 15-24.

[146] S. Patel, K.E. Boyd, J. Burns, Age, stability of the precorneal tear film and the refractive index of tears, Cont Lens Anterior Eye 23 (2000) 44-47.

[147] R. du Toit, P. Situ, T. Simpson, et al., The effects of six months of contact lens wear on the tear film, ocular surfaces, and symptoms of presbyopes, Optom Vis Sci 78 (2011) 455-462.

[148] M. Guillon, K. Dumbleton, P. Theodoratos, et al., Objective assessment of ocular surface response to contact Lens Wear in presbyopic contact Lens wearers of asian descent, Eye Contact Lens 44 (2018) 182-189.

[149] S.B. Hickson-Curran, R.L. Chalmers, L. Keay, et al., Patient-reported wearing experience from hydrogel daily disposable wearers older than 40 years from the TEMPO registry, Eye Contact Lens 43 (2017) 313-317.

[150] C.B. Nau, J. Harthan, E. Shorter, et al., Demographic characteristics and prescribing patterns of scleral Lens fitters: the SCOPE study, Eye Contact Lens 44 (Suppl. 1) (2018) S265-S272.

[151] S.J. Vincent, The rigid lens renaissance: a surge in sclerals, Cont Lens Anterior Eye 41 (2018) 139-143.

[152] M.M. Schornack, J. Pyle, S.V. Patel, Scleral lenses in the management of ocular surface disease, Ophthalmology 121 (2014) 1398-1405.

[153] E. van der Worp, D. Bornman, D.L. Ferreira, et al., Modern scleral contact lenses: a review, Cont Lens Anterior Eye 37 (2014) 240-250.

[154] S. La Porta Weber, R. Becco de Souza, Gomes JÁP, et al., The use of the esclera scleral contact Lens in the treatment of moderate to severe dry eye disease, Am J Ophthalmol 163 (2016) 167-173.

[155] E. Lafosse, D.M. Romín, J.J. Esteve-Taboada, et al., Comparison of the influence of corneo-scleral and scleral lenses on ocular surface and tear film metrics in a presbyopic population, Cont Lens Anterior Eye 41 (2018) 122-127.

[156] S.Z. Mian, J.S. Agranat, D.S. Jacobs, Prosthetic Replacement of the Ocular Surface Ecosystem (PROSE) Treatment for Complications After LASIK, Eye Contact Lens 42 (2016) 371-373.

[157] M.T.B. Nguyen, V. Thakrar, C.C. Chan, EyePrintPRO therapeutic scleral contact lens: indications and outcomes, Can J Ophthalmol 53 (2018) 66-70.

[158] T. Seiler, K. Koufala, G. Richter, Iatrogenic keratectasia after laser in situ keratomileusis, J Refract Surg 14 (1998) 312-317. 